\title{
Strain Mapping during In-situ Deformation using a High-Speed Electron Detector
}

C. Gammer ${ }^{1,2,3}$, J. Kacher ${ }^{1,2}$, J. Ciston ${ }^{1}$, C. Czarnik ${ }^{4}$, O.L. Warren ${ }^{5}$, A.M. Minor ${ }^{1,2}$

1. National Center for Electron Microscopy, Molecular Foundry, Lawrence Berkeley National Laboratory, Berkeley, CA 94720 USA

2. Department of Materials Science \& Engineering, University of California, Berkeley, CA 94720 USA

3. Physics of Nanostructured Materials, Faculty of Physics, University of Vienna, Austria

4. Gatan, Inc., Pleasanton, CA 94588 USA

5. Hysitron, Inc., Minneapolis, MN 55344 USA

Understanding the evolution of the local strain around individual defects during plastic deformation is of great importance for correlating defect structure with material properties. In-situ deformation in the TEM has provided great insight into the fundamental mechanisms occurring during deformation and many advances have been made in measuring the global plastic strain more accurately by using high precision transducers or image-correlation [1]. In addition, TEM has demonstrated the ability to measure strain fields around static dislocations [2], but measurements of the local elastic strain-field around individual defects during deformation are still lacking.

In the present work we show for the first time that strain mapping can be carried out during continuous in-situ deformation in a TEM at the nanometer scale. We use scanning nanobeam electron diffraction (NBED) for the strain mapping. Figure 1 shows the experimental setup. The converged beam rasters over the sample and on top of the annular dark-field (ADF) image, a full diffraction pattern is recorded for every probe position. A strain map can then be calculated by measuring changes in the lattice constants from the diffraction patterns [3]. In addition, virtual dark-field images can be computed from the diffraction maps [4]. The main practical limitation for this method is the speed of the electron detector. To overcome this limitation we used a Gatan K2 IS direct detection camera operating at a frame rate of $400 \mathrm{f} / \mathrm{s}$ and recorded a series of 22 nanodiffraction maps (64x64pixel) to calculate a time dependent local strain-map from this data. A Hysitron PI-95 picoindenter is used to pull an AlMg sample in tension. Figure 2 shows images extracted from the time-resolved dataset. The ADF image (cf. Fig 2a) shows complex diffraction contrasts from defects and misorientations in the sample. In the colorcoded strain map (cf. Fig 2b) two moving dislocations are visible. The results facilitate the comparison of the local and transient strains occurring around moving dislocations with the global strains measured with the picoindenter.

These results demonstrate how novel fast electron detectors enable real-time collection of diffraction maps at sufficient speeds to be combined with in-situ microscopy. The resulting datasets carry a large amount of information. Here we have shown how a time-resolved local strain-map can be extracted from a dataset acquired during in-situ deformation.

[1] Q. Yu, M. Legros and A.M. Minor, MRS Bulletin 40 (2015) p. 62.

[2] M. J. Hÿtch, E. Snoeck, and R. Kilaas, Ultramicroscopy 74 (1998) p. 131.

[3] V.B. Ozdol, C. Gammer, M.C. Sarahan and A.M. Minor, Microscopy and Microanalysis 20 S3 (2014) p. 1046.

[4] C. Gammer, V.B. Ozdol, C.H. Liebscher, and A.M. Minor, submitted to Ultramicroscopy. 
[5] The authors acknowledge support by the Austrian Science Fund (FWF):[J3397] and the Molecular Foundry, Lawrence Berkeley National Laboratory, which is supported by the U.S. Dept. of Energy under Contract \# DE-AC02-05CH11231.

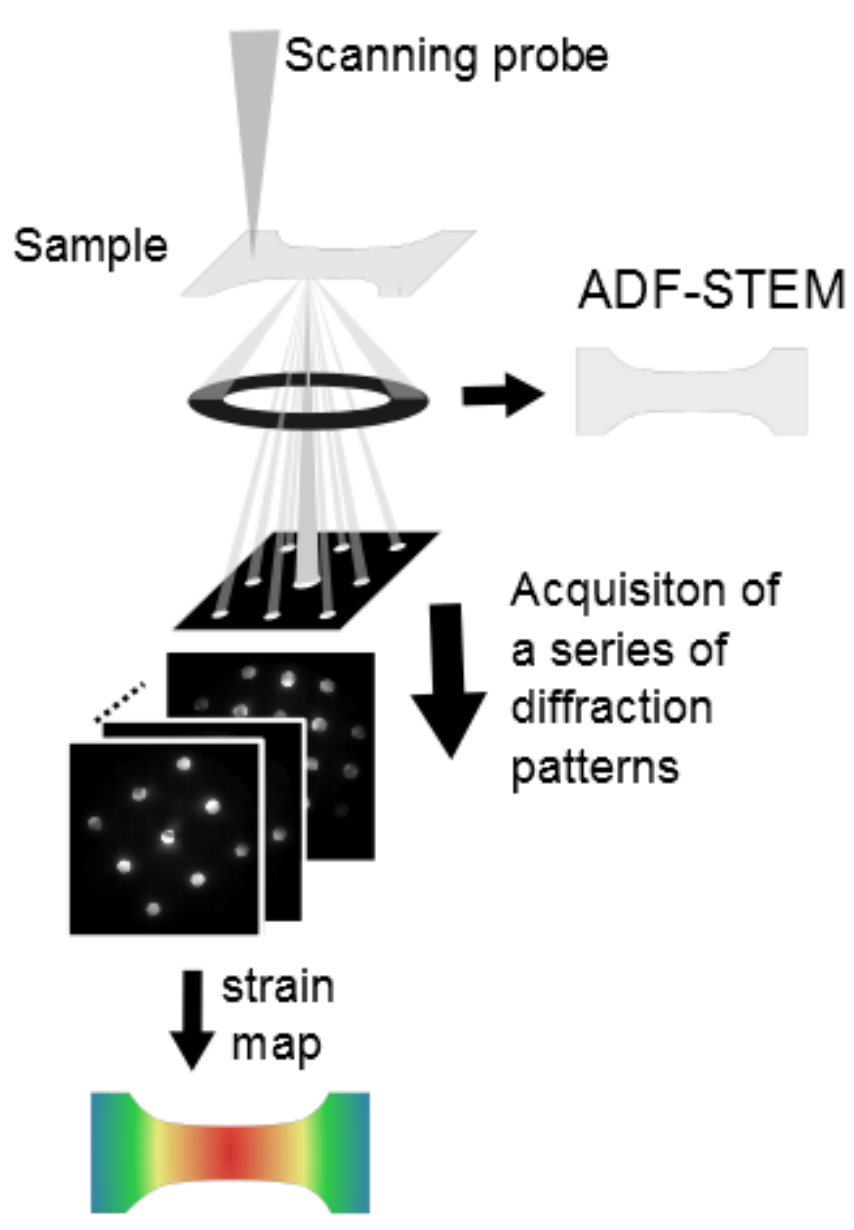

Figure 1. Setup used for nanodiffraction strainmapping. During acquisition of an ADF image in STEM mode, a diffraction pattern is acquired for every probe position. A strain map is calculated from the nanodiffraction map by measuring changes in the lattice constants from the diffraction patterns. (a)

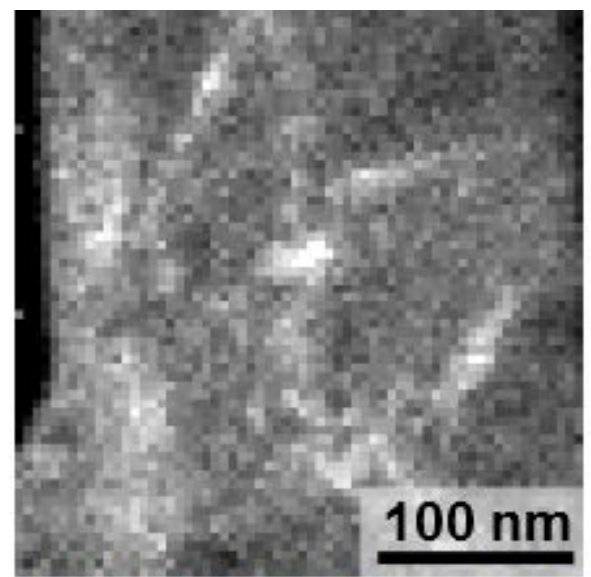

(b)

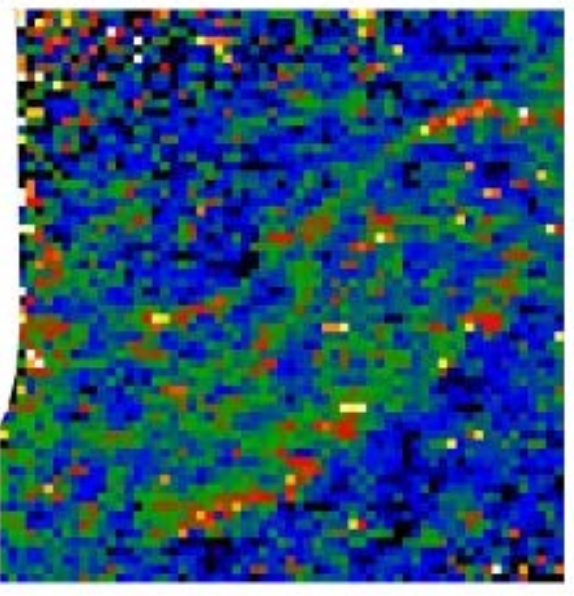

$-2 \%+4 \%$

Figure 2. Frame extracted from the in-situ strain mapping video. (a) ADF image acquired during deformation showing diffraction contrast due to defects and misorientations in the sample. (b) The corresponding color-coded strain map shows two moving dislocations. 